\title{
Unilateral Hyperlucent Lung Associated With Bronchial Atresia Mimicking Swyer-James Syndrome
}

\author{
Masaru Ando MD PhD, Eishi Miyazaki MD PhD, Hideaki Fujisaki MD, \\ Shin-ichi Nureki MD PhD, and Jun-ichi Kadota MD PhD
}

\section{Introduction}

Bronchial atresia is a rare pulmonary anomaly characterized by a blindly-terminating bronchus, mucoid impaction, and hyperinflation of the obstructed segment of the lung. ${ }^{1}$ Chest radiographic findings typically show mucoid impaction, segmental overinflation, and hypovascularity, although most patients do not have any respiratory symptoms. ${ }^{1,2}$ We herein present 2 cases of bronchial atresia with unilateral hyperlucent lung mimicking Swyer-James syndrome.

\section{Case Summary}

\section{Case 1}

A 28-y-old male experienced left chest pain while at work and was diagnosed with a left spontaneous pneumothorax at a nearby hospital. After $4 \mathrm{~d}$, he was referred to our department because the pneumothorax progressed during observation. His past medical and family history was unremarkable. He was $167 \mathrm{~cm}$ tall and weighed $48.2 \mathrm{~kg}$. His body temperature was $37.0^{\circ}$, his pulse was 75 beats $/ \mathrm{min}$ and regular, his blood pressure was 120/70 $\mathrm{mm} \mathrm{Hg}$, and his breathing frequency was 16 breaths/min on admission. Percussion and auscultation revealed decreased breath sounds over the left hemithorax, although no abnormalities in heart sounds were detected.

Drs Ando, Fujisaki, Nureki, and Kadota are affiliated with the Department of Respiratory Medicine and Infectious Diseases, and Dr Miyazaki is affiliated with the Center for Community Medicine, Oita University Faculty of Medicine, Oita, Japan.

The authors have disclosed no conflicts of interest.

Correspondence: Masaru Ando MD PhD, Department of Respiratory Medicine and Infectious Diseases, Oita University Faculty of Medicine, Idaigaoka 1-1, Hasama-machi, Yufu, Oita 879-5593, Japan. E-mail: mando@oita-u.ac.jp.

DOI: $10.4187 /$ respcare.03064
Intercostal tube drainage was immediately carried out on admission. A chest radiograph demonstrated a left pneumothorax with pleural adhesion of the upper field and slight deviation of the mediastinum toward the right (Fig. 1A). Chest high-resolution computed tomography (CT) performed after thoracic drainage demonstrated areas of low attenuation in the left upper lobe with a dilated bronchus and subpleural bullae (Fig. 1B). CT angiography revealed a narrowed left pulmonary artery and tributaries with undetectable peripheral branches. Ventilation-perfusion lung scintigraphy demonstrated a matched ventilation-perfusion defect in the upper and middle fields of the left lobe with marked air trapping during the washout phase (Fig. 2A). On bronchoscopy, neither bronchial atresia nor stenosis was observed.

These findings, including unilateral hyperlucent lung with decreased vascular markings, bronchial dilatation of the affected lung, and the matched ventilation-perfusion defect with air trapping during the washout phase, indicated a diagnosis of Swyer-James syndrome. Due to the persistent air leak, which lasted for $16 \mathrm{~d}$, lobectomy of the left upper lobe was performed. The resected lung demonstrated emphysematous changes in which part of the branch of the apicoposterior segmental bronchus was enlarged and contained a mucoid impaction. The dilated bronchus demonstrated a blind end (Fig. 3). Ultimately, the patient was diagnosed with lobar emphysema associated with bronchial atresia.

\section{Case 2}

A 36-y-old female first presented with abnormal chest radiographic findings $\sim 10 \mathrm{y}$ prior to the current presentation. She had been asymptomatic and had no significant medical history. She complained of a persistent dry cough without wheezing, and a long-acting bronchodilator was prescribed. Her symptoms remitted, and a chest radiograph showed hyperlucency of the left lung.

To identify the cause of the unilateral hyperlucent lung, the patient was referred to our department. Her past medical and family history was unremarkable. She was $151 \mathrm{~cm}$ 

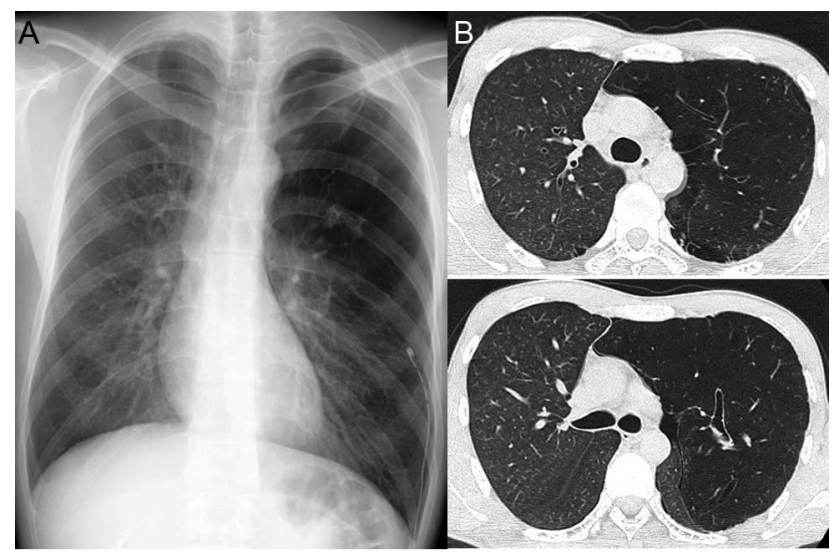

Fig. 1. A: Posteroanterior chest radiograph showing hypertranslucency of the left upper and middle fields with a marked reduction in the peripheral lung markings. B: High-resolution computed tomography scan showing emphysematous areas in the left upper lobe with a dilated bronchus and subpleural bullae.

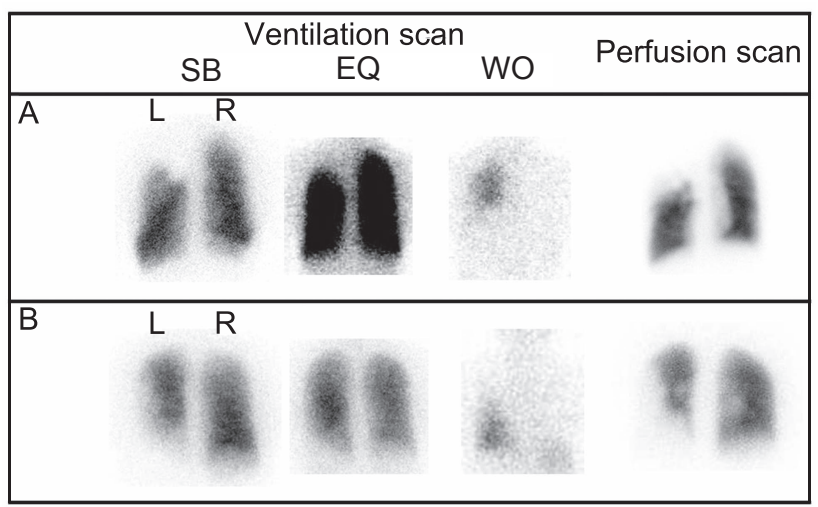

Fig. 2. Ventilation-perfusion lung scintigraphy. A: the matched ventilation-perfusion defect in the upper and middle fields of the left lobe of case 1. B: the matched ventilation-perfusion defect in the middle and lower fields of the left lobe of case 2. SB = singlebreath phase; $\mathrm{EQ}=$ equilibrium phase; $\mathrm{WO}=$ washout phase; $\mathrm{L}=$ left; $\mathrm{R}=$ right.

tall and weighed $57 \mathrm{~kg}$. Her body temperature was $36.4^{\circ}$, her pulse was 78 beats/min and regular, her blood pressure was $128 / 80 \mathrm{~mm} \mathrm{Hg}$, and her breathing frequency was 14 breaths/min. Percussion and auscultation revealed decreased breath sounds over the left hemithorax; however, no abnormalities in heart sounds were noted.

A chest radiograph demonstrated hyperlucency with bronchiectasis in the left middle and lower fields (Fig. 4A). The trachea had shifted slightly toward the right side; this shift was enhanced on a chest radiograph during the expiratory phase. Chest high-resolution CT demonstrated a large degree of hyperinflation in the left lower lobe with reduced pulmonary vessels and a mucocele. The lateral basal segmental bronchus (B9) of the left lung was dilated and did not communicate with the left inferior lobar bron-

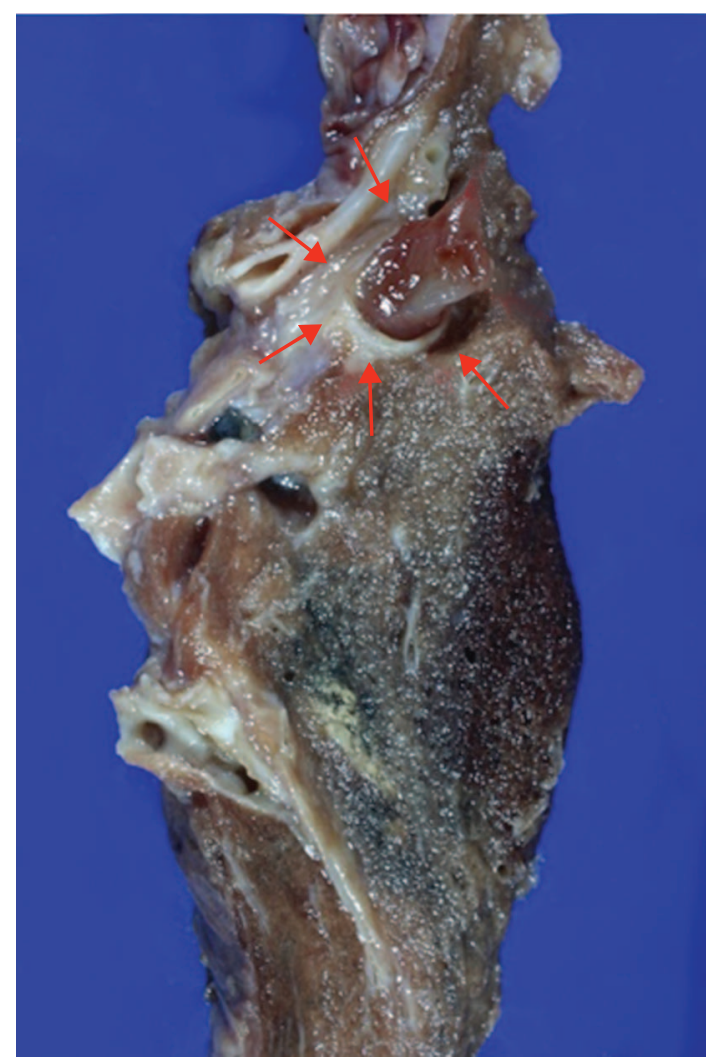

Fig. 3. The resected lung demonstrating mucoid impaction (arrows) with a blindly-terminating bronchus.

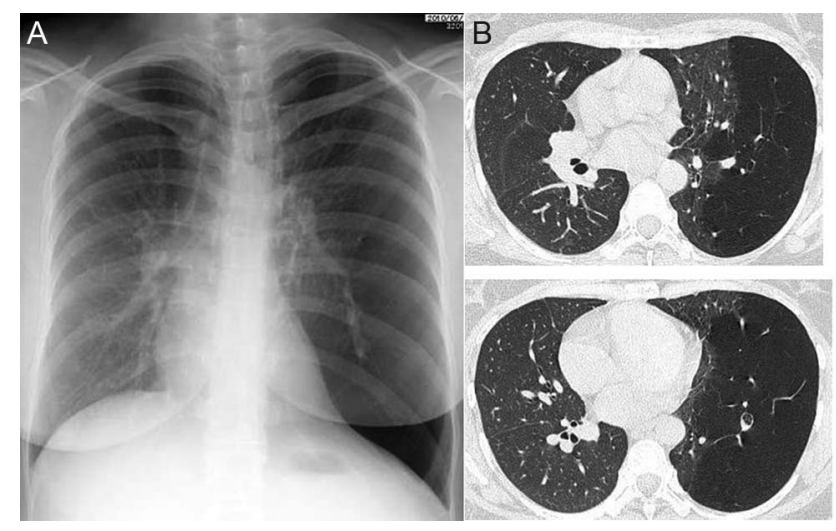

Fig. 4. A: Posteroanterior chest radiograph showing hypertranslucency of the left middle and lower fields with a marked reduction in the peripheral lung markings. B: High-resolution computed tomography scan showing a large degree of hyperinflation of the left lower lobe and reduced pulmonary vessels with dilated bronchioles and a few areas of mucoceles.

chus (Fig. 4B). Ventilation-perfusion lung scintigraphy demonstrated a matched ventilation-perfusion defect in the middle and lower fields of the left lobe with marked air trapping during the washout phase (see Fig. 2B). Bronchoscopy revealed complete obstruction of the orifice of 
the left B9. The patient was diagnosed with bronchial atresia of the lateral basal segment of the left lower lobe.

\section{Discussion}

Bronchial atresia is a rare congenital abnormality in which the bronchus lacks central airway communication. It is characterized by cystic, blindly-terminating, mucusfilled bronchoceles without connection to the main bronchial tree with normal subsequent generations of bronchi. Chest radiographic findings demonstrate round or lobulated perihilar, solid, or cystic masses as a sign of mucoid impaction. ${ }^{1}$ On the other hand, Swyer-James syndrome is characterized on chest radiographs by unilateral hyperlucent hemithorax formation with decreased vascular markings and on expiratory chest radiographs by air trapping. Moreover, bronchiectasis in either the ipsilateral or contralateral lung has been previously reported. ${ }^{3-5}$

In the 2 cases presented, a chest radiograph demonstrated unilateral hyperlucent lung with leftward displacement of the mediastinum, whereas chest CT revealed decreased vascular markings and bronchiectasis in emphysematous regions. Moreover, radionuclide ventilation scans showed delayed entry and washout in the affected regions, and the perfusion and ventilatory abnormalities were matched. These findings suggested a diagnosis of SwyerJames syndrome. In the pathogenesis of bronchial atresia, mucus continuously secreted from goblet cells and submucosal glands cannot pass through the blindly-terminating bronchus and accumulates, forming an area of a mucocele and causing progressive dilatation of the distal bronchus. In cases involving the transient spontaneous disappearance of a mucocele, as demonstrated in previous reports, ${ }^{6-8}$ the radiographic findings may masquerade as Swyer-James syndrome.

Bronchial atresia frequently demonstrates emphysematous changes in the affected peripheral lung, although it is a rare cause of unilateral hyperlucent lung. ${ }^{8}$ The emphysematous changes are thought to be due to the preserved ventilation through the pores of Kohn and canals of Lambert in the surrounding lung and bronchi and to the checkvalve mechanism. ${ }^{9}$ On the other hand, Swyer-James syndrome is an acquired condition characterized by a unilateral hyperlucent lung that usually develops during childhood as a sequel of postinfectious bronchitis obliterans. The main pathogenic event seems to be acute bronchitis with obliteration of small airways, leading to the dilation and destruction of the lung parenchyma with air trapping. ${ }^{10-12}$ To differentiate bronchial atresia from Swyer-James syndrome in patients with a unilateral hyperlucent lung on chest roentgenograms, the high-resolution CT finding of a normal distal bronchial branch pattern without a connection between the distal and central airways is essential.
Most patients with bronchial atresia do not have any respiratory symptoms, and the diagnosis is based on incidental findings. In the remaining $42 \%$ of cases, recurrent chest infections, coughing, and dyspnea are the most common symptoms. ${ }^{1}$ Bronchial atresia associated with pneumothorax formation is extremely rare in spite of overinflation of the involved lung parenchyma. To the best of our knowledge, this phenomenon has been demonstrated in only 3 reports. ${ }^{13-15}$ The underlying mechanism of spontaneous pneumothorax formation in patients with bronchial atresia is thought to be as follows: hyperinflation of the involved region, despite the presence of subsegmental bronchial obstruction, is caused by collateral air flow via the interalveolar pores of Kohn or bronchioloalveolar channels of Lambert and air trapping due to the check-valve mechanism. ${ }^{16}$ Moreover, the emphysematous changes induced by hyperinflation progress, results in the formation of bullae or blebs followed by the eventual development of a spontaneous pneumothorax due to rupture of the bullae. In fact, the presence of subpleural bullae or blebs in hyperinflated lung lesions has been observed in all cases of bronchial atresia followed by spontaneous pneumothorax formation.

In conclusion, we presented 2 cases of unilateral hyperlucent lung associated with bronchial atresia in which the radiographic findings mimicked those of Swyer-James syndrome. Although most patients with bronchial atresia do not have any respiratory symptoms, clinicians should recognize that the presence of subpleural bullae and blebs in emphysematous lesions predisposes patients with bronchial atresia to spontaneous pneumothorax formation.

\section{Teaching Points}

- The diagnosis of bronchial atresia is based on the presence of radiographic findings demonstrating a mucocele, occlusion of the central bronchus due to a mucocele, and emphysematous changes around the area of the mucocele.

- In our 2 cases, the chest radiographs demonstrated expanded emphysema with bronchiectasis due to the disappearance of the mucocele and decreased vascular markings. Moreover, the ventilation-perfusion scanning showed a matched ventilation-perfusion defect and air trapping during the washout phase on ventilation scanning. These findings may masquerade as Swyer-James syndrome.

- To differentiate bronchial atresia from Swyer-James syndrome, it is essential to determine whether there is a normal distal bronchial branch pattern without a connection to the distal and central airways by chest highresolution $\mathrm{CT}$. 


\section{Bronchial Atresia Mimicking Swyer-James Syndrome}

- Bronchial atresia should be considered in the differential diagnosis of a unilateral hyperlucent lung.

\section{ACKNOWLEDGMENTS}

We thank Dr Sigeo Hiroshige (Department of Respiratory Medicine, Oita University Faculty of Medicine, Oita, Japan), who was the attending physician, and Dr Mayumi Narahara (Department of Internal Medicine, Oita Kensei Hospital, Oita, Japan) and Dr Katsunori Sugisaki (Department of Pulmonary Disease, Nishibeppu National Hospital, Oita, Japan), who were the physicians of first contact before the subject was transferred to our hospital. We also thank Drs Masao Chujo, Michiyo Miyawaki, and Katsunobu Kawahara (Division of Respiratory Surgery, Oita University Faculty of Medicine) for performing the surgery; Dr Fumito Okada (Department of Radiology, Oita University Faculty of Medicine) for valuable assistance with the radiological findings; and Dr Ryu-ichi Takenaka (Department of Respiratory Medicine, Oita University Faculty of Medicine) and Mr Toshihide Kumamoto (Kyushu University of Nursing and Social Welfare, Kumamoto, Japan) for drafting the manuscript and providing advice about the important intellectual content.

\section{REFERENCES}

1. Jederlinic PJ, Sicilian LS, Baigelman W, Gaensler EA. Congenital bronchial atresia. A report of 4 cases and a review of the literature. Medicine 1987;66(1):73-83.

2. Matsushima H, Takayanagi N, Satoh M, Kurashima K, Kanauchi T, Hoshi T, Kanazawa M. Congenital bronchial atresia: radiologic findings in nine patients. J Comput Assist Tomogr 2002;26(5):860-864.

3. Marti-Bonmati L, Ruiz Perales F, Catala F, Mata JM, Calonge E. CT findings in Swyer-James syndrome. Radiology 1989;172(2):477-480.

4. Ohri SK, Rutty G, Fountain SW. Acquired segmental emphysema: the enlarging spectrum of Swyer-James/Macleod's syndrome. Ann Thorac Surg 1993;56(1):120-124.
5. Moore AD, Godwin JD, Dietrich PA, Verschakelen JA, Henderson WR Jr. Swyer-James syndrome: CT findings in eight patients. AJR 1992;158(6):1211-1215.

6. Miyahara N, Eda R, Makihara S, Maeda T, Aoe K, Kunichika N, et al. Bronchial atresia with transient spontaneous disappearance of a mucocele. Intern Med 1999;38(12):974-978.

7. Oh KS, Dorst JP, White JJ, Haller JA Jr, Johnson BA, Byrne WD. The syndrome of bronchial atresia or stenosis with mucocele and focal hyperinflation of the lung. Johns Hopkins Med J 1976;138(2): 48-53.

8. Genereux GP. Bronchial atresia: a rare cause of unilateral lung hypertranslucency. J Can Assoc Radiol 1971;22(1):71-82.

9. Meng RL, Jensik RJ, Faber LP, Matthew GR, Kittle CF. Bronchial atresia. Ann Thorac Surg 1978;25(3):184-192.

10. Hardy KA, Schidlow DV, Zaeri N. Obliterative bronchiolitis in children. Chest 1988;93(3):460-466.

11. Kogutt MS, Swischuk LE, Goldblum R. Swyer-James syndrome (unilateral hyperlucent lung) in children. Am J Dis Child 1973; 125(4):614-618.

12. Daniel TL, Woodring JH, Vandiviere HM, Wilson HD. Swyer-James syndrome-unilateral hyperlucent lung syndrome. A case report and review. Clin Pediatr (Phila) 1984;23(7):393-397.

13. Kameyama K, Okumura N, Kokado Y, Miyoshi K, Matsuoka T, Nakagawa T. Congenital bronchial atresia associated with spontaneous pneumothorax. Ann Thorac Surg 2006;82(4):1497-1499.

14. Yoon YH, Son KH, Kim JT, Baek WK, Kim KH, Lee KH, Han HS. Bronchial atresia associated with spontaneous pneumothorax: report of a case. J Korean Med Sci 2004;19(1):142-144.

15. Berkman N, Bar-Ziv J, Breuer R. Recurrent spontaneous pneumothorax associated with bronchial atresia. Respir Med 1996;90(5): 307-309.

16. Culiner MM, Reich SB. Collateral ventilation and localized emphysema. Am J Roentgenol Radium Ther Nucl Med 1961;85(2):246252. 